\title{
Erratum: Regularization of instabilities in gravity theories [Phys. Rev. D 97, 024008 (2018)]
}

\author{
Fethi M. Ramazanoğlu
}

(Received 14 March 2019; published 28 March 2019)

DOI: 10.1103/PhysRevD.99.069905

The equations of motion for ghost-based spontaneous growth are missing some terms in this published paper. The two main modifications are that Eq. (4) should read

$$
\nabla_{\mu}\left[\left(-8 \pi \tilde{T} A_{\partial}^{4} \alpha_{\partial}+1\right) \nabla^{\mu} \phi\right]=m_{\phi}^{2} \phi
$$

and Eq. (12) should read

$$
\nabla_{\rho}\left[\left(-8 \pi \tilde{T} A_{F}^{4} \alpha_{F}+1\right) F^{\rho \mu}\right]=m_{X}^{2} X^{\mu}
$$

Note that the terms lacking in the published version, $\nabla^{\mu} \phi \nabla_{\mu}\left(\tilde{T} A_{\eta}^{4} \alpha_{\eta}\right)$ and $F^{\rho \mu} \nabla_{\rho}\left(\tilde{T} A_{F}^{4} \alpha_{F}\right)$, do not appear in the principal part of the linearized equations. Consequently, they do not affect our main conclusions about the existence of ghost-based spontaneous growth for scalars or vectors.

The correction to $\phi$ equation above is also reflected in three other equations. First, Eq. (5) changes to

$$
\left(-4 \pi A_{\partial}^{4} \beta_{\partial} \tilde{T}+1\right) \square_{g} \phi \approx m_{\phi}^{2} \phi+4 \pi A_{\partial}^{4} \beta_{\partial} \nabla_{\mu} \tilde{T} \nabla^{\mu} \phi .
$$

Second, Eq. (6) changes to

$$
g^{t t} \partial_{t}^{2} \phi+\cdots \approx\left(-4 \pi A_{\partial}^{4} \beta_{\partial} \tilde{T}+1\right) m_{\phi}^{2} \phi .
$$

Third, Eq. (8), the modified Tolman-Oppenheimer-Volkoff (TOV) equation, changes for $\phi$ as

$$
\begin{aligned}
\phi^{\prime} & =\chi / \sqrt{1-x} \\
C_{\chi} \chi^{\prime} & =-\left[C_{\nu} \nu^{\prime} / 2+2 / r\right] \chi+\gamma^{-1} m_{\phi}^{2} \phi / \sqrt{1-x},
\end{aligned}
$$

where

$$
\begin{aligned}
& C_{\chi}=1-16 \pi A_{\partial}^{4} \gamma^{-1} \chi^{2} \times\left[\left(4 \alpha_{\partial}^{2}+\alpha_{\partial}^{\prime}\right)(-\tilde{\rho}+3 \tilde{p})-\alpha_{\partial}^{2}\left(-\tilde{\rho}^{\prime}+3\right)(\tilde{\rho}+\tilde{p})\right], \\
& C_{\nu}=1+8 \pi A_{\partial}^{4} \alpha_{\partial} \gamma^{-1}\left(-\tilde{\rho}^{\prime}+3\right)(\tilde{\rho}+\tilde{p})
\end{aligned}
$$

with $\tilde{\rho}^{\prime} \equiv d \tilde{\rho} / d \tilde{p}$ and $\alpha_{\partial}^{\prime} \equiv d \alpha_{\partial} / d \eta$.

The neutron star in Fig. 1 is based on the original erroneous modified TOV equations and hence is not a valid nonlinear solution. Nevertheless, it qualitatively reflects all the main features of a spontaneously scalarized star through a ghostlike instability, such as the cusp in the scalar field which still occurs for the corrected equations.

There is also a minor typo in Eq. (11) where the correct definition is $\eta_{F}=F_{\mu \nu} F^{\mu \nu} / 2$, i.e., the $1 / 2$ factor is missing in the original published version. 